\title{
Analysis of the Fractal Koch Method in Computer Games Development
}

\author{
Farhad Soleimanian Gharehchopogh $^{1}$, Isa Maleki ${ }^{2}$, Sahar Sadouni $^{3}$ \\ ${ }^{1}$ Department of Computer Engineering, Urmia Branch, Islamic Azad University, \\ Urmia, Iran \\ ${ }^{2}$ Department of Computer Engineering, Dehdasht Branch, Islamic Azad University, \\ Dehdasht, Iran \\ ${ }^{3}$ Department of Computer Engineering, Science and Research Branch, Islamic Azad \\ University, West Azerbaijan, Iran
}

\begin{abstract}
Lately according to the software and hardware progress, the development of the computer games has been so much. As the development of the computer games is one of the most favorite projects of computer sciences. A requirement for development of the computer games is the computer graphics programming science and the mathematical calculations. A calculation method is fractal Koch which uses the selfsimilarity specifications of itself. Formation of fractals takes place by the repetitions, so it is possible to transform a simple geometric shape to a more complex one and use the fractal capabilities to develop and produce the computer games. So, we have tried to use the OpenGL graphic functions in VC++6.0 and review the application of fractal Koch in development of the computer games.
\end{abstract}

\section{KEYWORDS}

Development of Computer Games, Graphic Programming, Fractal Koch, OpenGL

\section{INTRODUCTION}

The computer games are one of the most powerful and widest branches of computer graphics. Computer graphics is used widely of which the use of different two-dimensional (2D) and threedimensional (3D) technics in designing and development of the computer games are the most general ones $[1,2]$. Development of the computer games in the late decades from the 3D graphic views have been noticed clearly. The computer games have been developed using the graphic software for game designing and the graphic programming environments. So, development of the computer games is a kind of software development in which the computer games are produced [3, 4]. The game development software's one of the most complex ones as they work with different elements like editors, compilers and the peripheral supplies. The design and the development of the computers games could be done by the editors and the developed programming environments which make the development of the computer games easier and fast.

Nowadays the programming languages include diverse libraries of the classes and the Application Program Interface (API) to develop the programs which need mastering. Using the programming libraries OpenGL (Open Graphic Library), makes development of the computer games in 2D and 3D fast [5]. OpenGL functions for computer games are very powerful for 
development of the computer games and provide facilities like structuring 3D models, lighting and the construction of the shapes for the computer games producers to make the output of the games look like as real [6]. OpenGL functions provide a programming interface for 2D and 3D computer games. OpenGL is a modeling and 3D graphic in nature which is very fast and transferrable. Using the OpenGL it is possible to design high quality and high graphic 3D pictures. OpenGL is a library in $\mathrm{VC}++$ programming environment which provides some pre packed functions for creating the computer games.

Fractals use self-similarity and repetitions to create shapes in unlike the Euclid shapes [7]-[8]. The fractal shapes are erratic and also the disorder rate is fixed for any scale. It is possible to use the fractals as one of the most important models in developing the computer games [9]-[10]. In computer games to have pictures of high quality, any point of the pictures must be registered accurately and this uses a high volume of the memory. So, using the fractals is a good method to reduce the volume of the images in computer games. Fractals are used for solving the complex problems and in different scientific and engineering fields like computer graphics [11], image processing $[12,13]$ and communication [14].

The structure of the paper is as follows: in Section 2, we have introduce the related works; in Section 3, we describe the computer games, in Section 4, we describe the fractals and their application in development of the computer games; in Section 5, the results of simulation are presented and at finally in the Section 6, conclusion and future works is presented.

\section{RELATED WORKS}

Nowadays, the development and designing the computer games is done using the game engines and the different technologies of programming in 2D and 3D models. So, it is possible to say that computer graphics has made many changes in calculation and graphic methods. This branch is one of the widest and the most applied sciences in development of the computer games. Computer graphic has made the designers of the computer games simulate the games with no need for calculations via game structuring software. In this case we point out some works done this field.

$\mathrm{R}$. Li [6] has used the OpenGL technology in developed programming environment of $\mathrm{VC}++$ to develop the computer games. In this paper model of 3D and lighting of the shapes in computer games are emphasized. To have high quality computer games and to evaluate the colors of the images, it is possible to use OpenGL and transform the graphic states of the images to the bit vectors and present the images with high quality. Of the other specifications of 3D shapes, the capacity of absorbing light and maintaining it must be pointed. Without lighting, nothing is visible and if the shapes are visible they are presented in 2D. When we draw the shapes of a game, we must consider the lighting of them because even the best 3D shapes will not be considerable without light. The results of the paper show that the OpenGL technology is capable of creating 2D and 3D images. Researchers [15] have discussed the challenges of software engineering. They have studied the subject of 3D models in computer games. They have described that one of the debates on $3 \mathrm{D}$ graphic is the texture. Texture is the $2 \mathrm{D}$ image which is mapped in a special way on the surface and the 3D things to make it seem real. One of the other factors of 3D shapes creation is the lighting. In lighting calculations, the angel of the source light and the surface of the things must be finely adjusted. They have also emphasized that to make the development procedure of the computer games better, the software engineering patterns must be used. 
Researchers in [16] have used the OpenGL technology to structure and develop the specifications of the 3D images. In development of the computer games, the most important factor is the images. Of the most important specifications of 3D graphic done on the images are reading, presenting, location change, rotation and filtering the images. In OpenGL technology, the 3D graphic is based on vertex. In fact using the vertex, it is possible to create the shapes and it is possible to transfer them to the 3D environment and create the moving objects easily. They have also cited that in 3D graphic, matrix are used to create the shapes. So, it is possible to use them and make many transformations on the 3D objects. Transfer, rotations, size change and the reflection on a surface (for example to make shadow) could be implemented by matrix operations. Also they have examined the OpenGL functions for creation of 3D objects. Researchers [17] have discussed the 3D models in $\mathrm{VC}++6.0$ programming environment using the OpenGL. They have cited that OpenGL is an advanced API and also a powerful one for 3D graphic designs. One of the most used techniques in animation is the texture. Using the texture technique it is possible to transform the shapes into many forms. The results of the examinations show that the OpenGL s very efficient in forming the shapes and the 3D models.

D. Fang [18] is discussed 3D simulation of terrain according fractal. One of the most advance techniques in 3D graphic is the ability of making any kind of terrain and the natural environments. It is possible to simulate mountains, plains, hills and any other non-equal surfaces using the fractal. In this paper, the fractal theory and fractal Brownian motion have been studied. And the 3D objects like cloud and sky are created using fractal. So, now the graphic capabilities have made many changes in development of the computer games which are the general part of the computer games industry. It also cited in this paper that the 2D and 3D models, all utilize the graphic capabilities to logically balance the relationship between the quality of the images and the speed of execution of the games. The results of the simulation in this paper are got from $\mathrm{VC}++6.0$ programming environment and using the OpenGL technology. The reference [19] has plotted the natural shapes using the fractal geometry. This reference has plotted the 3D objects in VC++6.0 programming environment using the OpenGL. The fractal geometry makes the plotting the natural shapes like sky and the mountains possible. So, using fractal, it is possible to simulate the real world phenomena.

In [20] have discussed the 3D computer games development using the programming and game engines fields. In this paper it is described that the programming languages have some capabilities and also some limitations each, and any of them are used in any special field and some are more dynamic in computer games world. Also the use of API is a big change in structuring the computer games and have led to the computer graphic and now the computer games are directly or indirectly (via the game engine) use the programming interface. Also, in the results section, some samples of games are designed using the game engine and programming functions.

\section{COMPuTER GAMES}

Development of the computer games using the game engines and the programming environments has widened the advanced 2D and 3D of the games. So, the development of the computer games using the advanced game making tools has made the game making procedure easier for the programmers and the designers. In development of the computer games it is very important to utilize the tools. These tools include knowledge and the experiment of the people for development of the computer games. One of the very important tools in computer games is 
programming the graphic environments. The computer games are potential in graphic, simulation and imaging and are considered by many users. Lately the progress of the 3D games has been one of the poles of game making industry [21]. So, the computer game industry is a very high profit making one all over the world and the all day's progress of it has made many companies to design the games which are powerful in multi-dimensional graphic.

The process of making and developing the computer games was first done by the elementary programmers. But these activities have changed to a master level since 1970. And the game developing teams started to work professionally on the game designing [21]. So, the process of the completion and the complexity of the designing the computer games has developed and any part of game developments has been done by the masters of that part. Now the game engines are one of the most complex computer programs in the teams of developing the computer games. Game engines are in fact the software element and the main element of any computer game which produce 2D and 3D graphic environments [22]. The game engine uses the basic technology and makes the development of the games easier and provides a possibility to execute the games on the game councils and the computers.

In development of the computer games of $2 \mathrm{D}$ and $3 \mathrm{D}$, the factors like lighting, texture and rendering are very effective. One of the most important specifications for making the $2 \mathrm{D}$ and $3 \mathrm{D}$ computer games more real is shadow [23]. Shadows are in direct relation to the light and are divided into three groups. In the first type, the shadow is created by the fixed shape on the smooth surface. In the second type the shadow is created on the rendered images. This is the fast way of shadow making. But it is possible to use it to shadow on the smooth surface. The most flexible shadows are the volume shadows. Volume shadows can be created on any surface and they also can make shadow on the moving things. This shadow is the most complex and the slowest kind of shadows. One of the other processes taking place working with the images, is the lighting of pats of the images. This technique is very applicable in here dimensional graphics. It is also possible to combine the colored elements of the images and map texture on the shapes of the game environment. One of the characteristics of the game engines is the Rendering which creates the 3D capability. Rendering is a capability which transforms the basic graphic shapes to the images [24]. This kind of the engines are often called rendering Engine or 3D engines. So, engines create capabilities required for 3D images for the developers of the computer games. The 3D or rendering engines are structured according to the graphic technologies like direct 3D or OpenGL which cause hardware dependency from the graphic cards. So, one of the requirements of developing the computer games is the knowledge in the wide area of science like computer graphic, image processing and computer calculations. The development of the computer games and computer graphic needs mastering in mathematics and computer algorithms.

Designing and programming the computer games are of the most important activities in computer game industry which investigate on the professional teams of game creation So, the structuring the computer game seems like development of the software systems for development of the very advanced samples may a team of professionals in designing, animation and programming work for many years. In computer games industry, the $3 \mathrm{D}$ graphic models are faced in many action designing and have been developed by progress of the tools of this industry [25]. The computer games are not only the tools for business activities, but also are used in many training fields and the computer industry is progressing in training sections [26]. So, it is possible to develop and design the 2D and 3D games in a way that they will be used for training in programming, different learning strategies, increasing the concentration, competitiveness and increasing the different professions. 


\section{Fractal}

The fractal geometry was first presented by Mandelbrot [27]. In fractal geometry, it is possible to create different models of fractal and model the different shapes and using the fractal geometry, the accurate status and the irregular shapes could be combined and the complex shapes are created [28]. The fractal geometry describes the things which are similar or symmetric. So, when the things are enlarged, an accurate similarity exists among their elements and this similarity continues in a limited or unlimited end [29]. One of the most famous fractals is called Koch, which is designed by Helge von Koch [8]. In this fractal a segment of unit length is considered and then it is divided into three parts. The middle part is removed and the others are considered as the sides of equilateral and the procedure is repeated [30]. If this is done for any of the segments and is continued until no end, fractal Koch is shaped. Shaping stages of fractal Koch are showed in Figure (1).

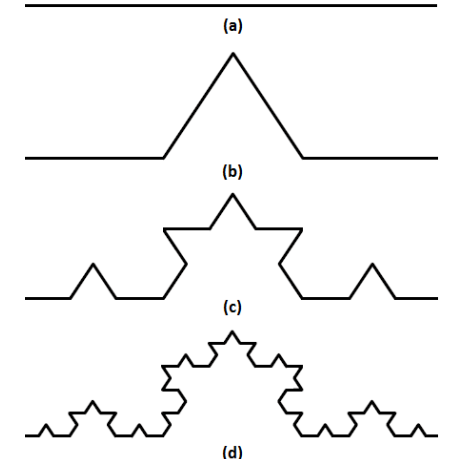

Figure 1. The Fractal Koch Formation Stages

Fractal Koch is a kind of self-simulating fractal. So, the fractal Koch of a shape or a geometric pattern of same parts is a total shape. An interesting point in studying the fractals is the dimension of them. So, each shape in fractal is the ratio of the logarithm of the number of the shapes of the self-similarity to the enlargement factor. The fractal dimension is calculated according to the equation (1) [8].

$$
D=\frac{\log N}{\log S}
$$

In equation (1), $N$ is the number of very fine parts which form the main shape. Value $S$ compares the small parts with the larger shape. And $D$ is the fractal dimension of the shapes. According to the equation (1), the fractal dimension $s$ identified according to the equation (2).

$$
D=\frac{\log N}{\log S}=\frac{\log 4}{\log 3}=1.2691
$$

Figure (2) shows the quasi code of fractal Koch.

\footnotetext{
1. Initialize Parameters

$$
\mathrm{N}=4
$$$$
\mathrm{S}=3
$$$$
\text { 2. Do }
$$

Calculating Fractal Dimension

Draw Koch Curve

3. Loop until (End Iterations)
} 
Figure 2. The Quasi Code of Fractal Koch

Figure (3) shows fractal Koch. As it can be seen, the output holds regular swings in all repetitions and all fractal dimensions are in similar size. The harmonization of fractal Koch and the scale increase procedure is done in a regular and logic method. So, fractal Koch holds dimension scaling from structure point of view and this causes using fractal Koch and creates similar or different shapes.

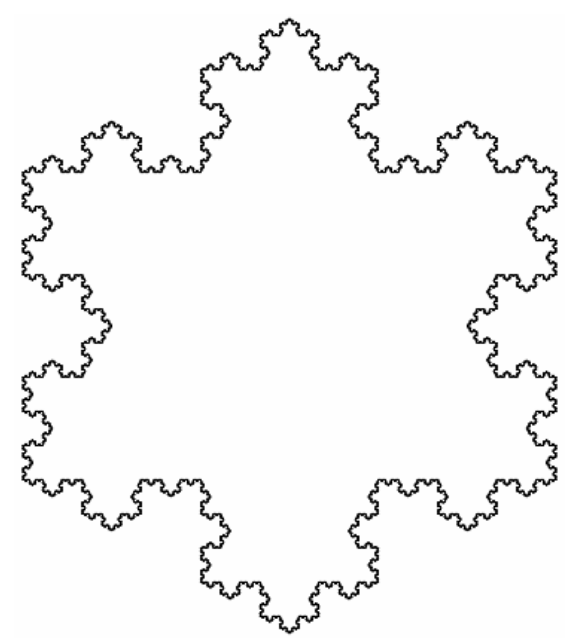

Figure 3. Fractal Koch

Three techniques for creation of the fractal shapes are [27, 7]:

Recursive Fractals: in these fractals, the fractal dimensions are formed by a recursive relationship and are presented similar in different scales.

Repetitive Fractals: These fractals hold a fixed replacement regulation and are presented similar in different scales.

Random Fractals: These fractals are made of random repetitions. Random fractals are the fractals which are in some kind, self-similar but are not completely self-similar or semi selfsimilar.

\subsection{Application of Fractal Koch in Development of Computer Games}

In development of the computer games, it is important to be near to the reality for the producers of the computer games. The graphic of the computer games is very important in the realness of the portraits, advanced effects and making real the environment of the games. In development of the computer games, when the deep colors and accurate plotting is done by the high quality graphic, the favorite outcomes are resulted. The effective graphics like the reflection of like light surfaces and the different patterns (like clouds, ground) are very important in the realness of the computer games. So, it is possible to adjust the dimension, location, speed and color to create total real shapes in $2 \mathrm{D}$ and $3 \mathrm{D}$ pages. The other technique of the computer games is the animation of the textures. It is possible to use fractal to create moving shapes in 3D graphic. And also it is possible to use fractal on the texture to reshape the image to a favorite shape. In general the fractals are the controlled shapes and are based on the scaling and distribution 
procedures. Development and the distribution of the fractal shapes use the simple fractal model to create the multi fractal technology and it is possible to combine them and create different shapes. To go high in efficiency and the speed of the computer games and also to decrease the use of the images in development of the computer games, fractal Koch is used. Figure (4) shows the development of the computer games using fractal Koch [27] and OpenGL [5].

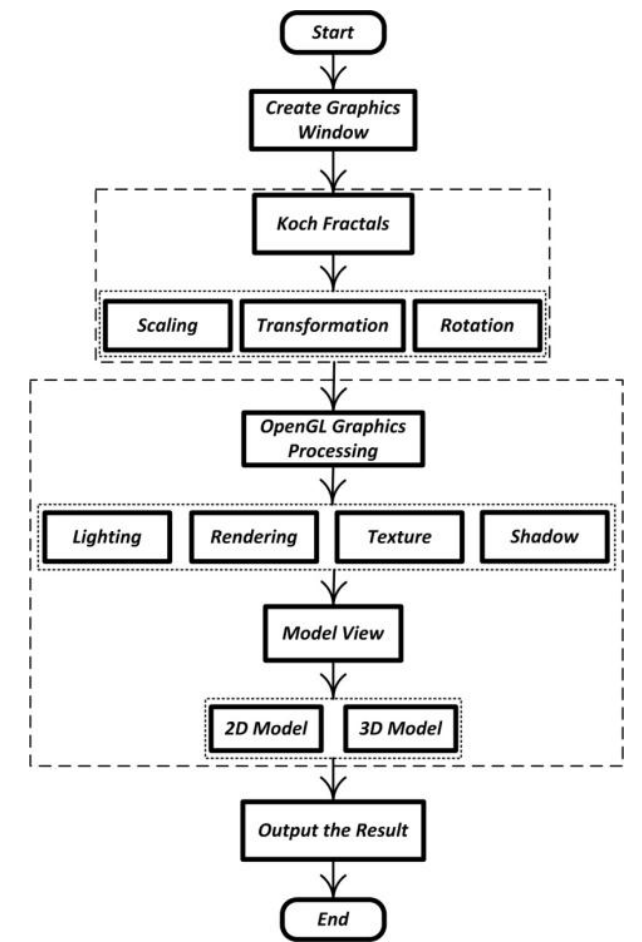

Figure 4. Development of the Computer Games using the Fractal Koch and OpenGL

In Figure (5) the quasi code used for development of the computer games using fractal Koch is showed:

\begin{tabular}{|l|}
\hline 1. Start \\
2. Create Environment Game \\
3. Loop \\
3.1. Koch Fractal \\
Draw Graphics Shape \\
• Scaling \\
• Transformation \\
• Rotation \\
4. Until (end iterations) \\
5. OpenGL graphics library \\
Function Calls \\
- Lighting \\
• Rendering \\
• Texture \\
• Shadow \\
6. Display Model \\
•2D Model \\
•3D Model \\
7. Output the Result \\
8. End
\end{tabular}


Figure 5. The Quasi Code for Development of the Computer Games using Fractal Koch.

Most of the 3D shapes used in computer games are formed from many polyhedral. Any polyhedral includes some pinnacles and using fractal it is possible to plot the polyhedral to 2D and $3 \mathrm{D}$ shapes. So, the most important thing in creating the computer games of $2 \mathrm{D}$ and $3 \mathrm{D}$ is to plot to shapes of sides and angles. After plotting the multi dimension models based on the fractal geometry, it is possible to transform the shapes to the set of the patterns to present in the game environment. As a result, the most common way to plot the polyhedral shapes and the multi-dimensional images, is to use the fractal. It is possible to use the fractals and create moving images in 2D and 3D game environments. So, fractal holds high capacity and is a good technique for development of the computer games. Using the fractal, it is possible to create regular and irregular shapes of games. Using the fractal equations it is possible to shape ground, cloud and fire.

\section{RESUltS OF SimULATION}

Modeling and creation of the graphic environment are two important stages of computer graphic. The main goal of modeling is to conform the shapes to the multi-dimensional environments. To implement and simulate the development of the computer games, the fractal Koch of VC++6.0 and OpenGL functions are used.

To image the realities in the computer games is an important specification $\mathrm{n}$ computer games. So, the graphic of the games depends on the items like the implementation. The fractal graphic shapes are often formed of the geometric shapes and the continuous points and the shape which images the fractal is in real the combination of the mathematical coordinates and the relation between the repetitions of the fractal shapes. In fact, the fractal is a kind of graphic in which the images are formed according to the relation of the points. A specification of the graphic fractal shapes is depicting the images. The formation of the graphic shapes using the fractals in fact to put the geometric shapes on each any of which are produced by the size change, transfer and rotation. So, to create the graphic images and the conformation of them a real shape, it is necessary to place the fractal Koch shapes on a $2 \mathrm{D}$ page and operate on them to create the $3 \mathrm{D}$ shape of them. And also to blazon the fractal shapes, it is possible to use the OpenGL functions to deepen their color. To show the efficiency of the fractal Koch in development of the computer games, the 2D and 3D cloud and fire are simulated. 
International Journal of Computer Graphics \& Animation (IJCGA) Vol.4, No.1, January 2014

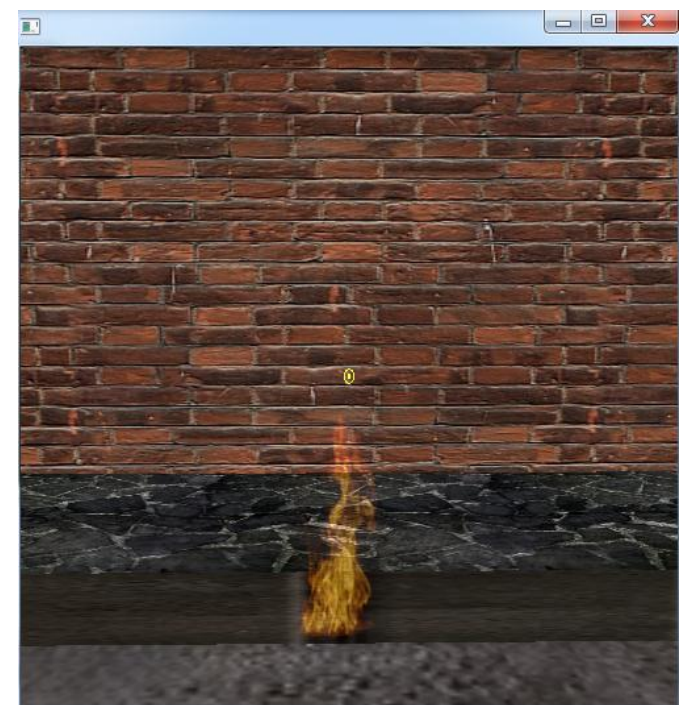

Figure 6. The Display 2D Model of the Fire Make

Fractals are the elements the space form of which is not regular. It is possible to simulate the natural phenomena using the irregularity of them in different scales. As it is seen in Figure (6), the $2 \mathrm{D}$ fire is created by the repetition of fractal Koch. So, the fire movement is simulated by the fractal Koch dynamic structure.

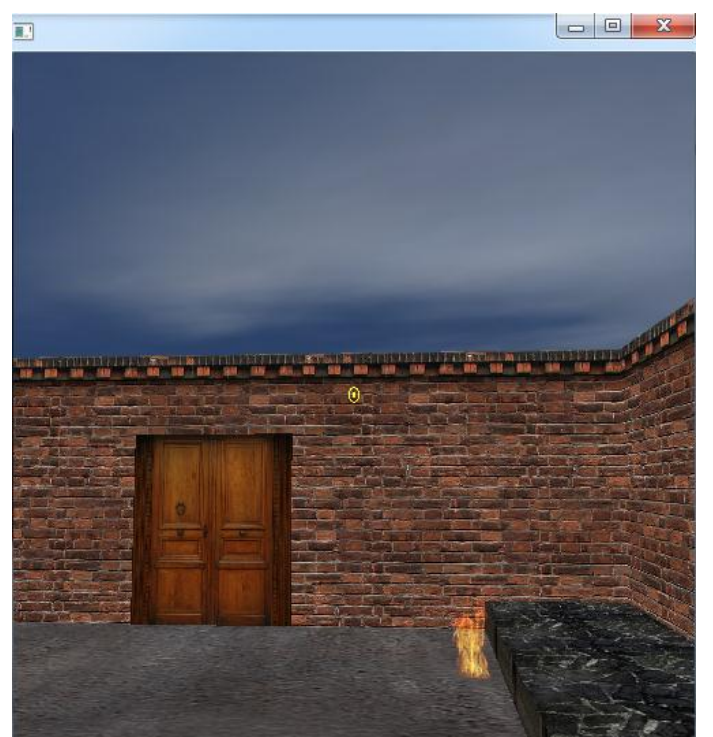

Figure 7. The Display 2D Model of the Cloud Make

In Figure (7) the 2D model of the cloud is showed using of the fractal Koch. As the clouds are shaped irregularly, the fractal Koch could be used to create the different cloud shape. It is also possible to create real and fiction shapes by more repetitions of fractal equations. 


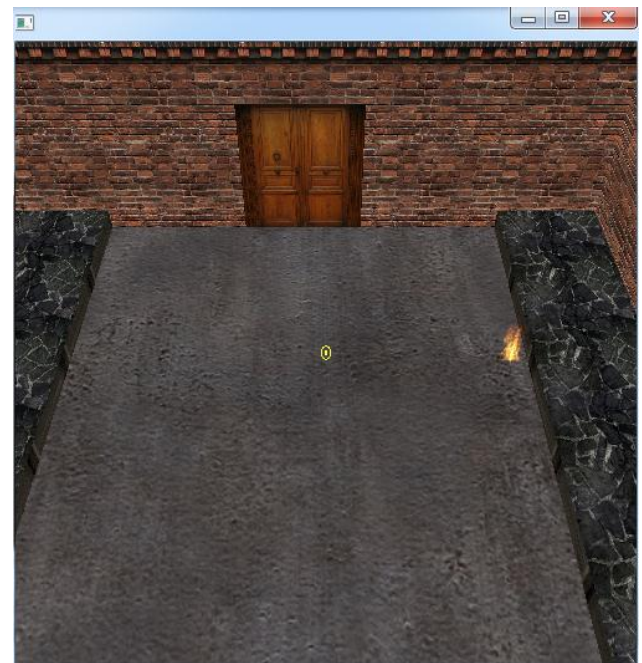

Figure 8. The Display 3D Model of the Fire Make

In Figure (8), 3D model of the fire is showed. To see the 3D fire, the OpenGL functions are used to make the camera view in 3D and the environment could be simulated from any side.

\section{CONCLUSION AND FUtURE WORKS}

Nowadays there are many 2D and 3D graphic software. Maybe the most general use of computer 3D graphic is the computer games. Now it is possible to produce games using the programming technique and the game engines which enjoy high graphic quality and charm. So it is possible to create geometric and compound shapes in programming environments using the fractal mathematics models and develop the games fast and use the OpenGL techniques to present the fractal shapes in 2D and 3D display. So, it could be said that the field of computer games develop progresses day by day. We hope that in future will be able design programs and computer games which advanced image effects and favorite graphic in terms of multidimensional images for the users create.

\section{REFERENCES}

[1] Ch. Seddon, “OpenGL Game Development”, pp. 411-421, Wordware Publishing, United States of America, 2005.

[2] B. Chen, H.H. Cheng, "Interpretive OpenGL for Computer Graphics", Computers \& Graphics, Vol. 29, pp. 331-339, Elsevier Ltd, 2005.

[3] P. Gestwicki, S. Fu-Shing, B. Dean, "Teaching Game Design and Game Programming Through Interdisciplinary Courses", Journal of Computing Sciences in Colleges, Vol. 24, No. 1, pp. 110-115, 2008.

[4] A. Rollings, D. Morris, "Game Architecture and Design", New Riders Publishing, 2003.

[5] L. Benstead, "Beginning OpenGL Game Programming”, Course Technology PTR, March 2009.

[6] R. Li, "Study on the Virtual Simulation of Flight Environment Based on OpenGL", Computer and Information Science, Vol. 2, No. 2, May 2009.

[7] K. Falconer, "Fractal Geometry: Mathematical Foundations and Applications", Wiley, Nov 2003. 
[8] P. Horvath, P. Smid, I. Vaskova, M. Hrabovsky, "Koch Fractals in Physical Optics and their Fraunhofer Diffraction Patterns", pp. 206-213, Elsevier GmbH, 2010.

[9] T. Martyn, "Realistic rendering 3D IFS fractals in real-time with graphics accelerators", Computers \& Graphics, pp. 167-175, Elsevier Ltd, 2010.

[10] A. Norton, "Generation and display of geometric fractals in 3-D”, Computer Graphics, Vol. 16, No. 3, 1982.

[11] J. Helmstedt, "A New Method of Constructing Fractals and Other Graphics", The Mathematica Journal, pp. 1-26, Wolfram Media, 2011.

[12] M. Kamran, A.I. Sipra, M. Nadeem, "A Novel Domain Optimization Technique in Fractal Image Compression", IEEE Proceedings of the 8th World Congress on Intelligent Control and Automation, pp. 994-999, 2010.

[13] S.V. Veenadevi, A.G. Ananth, "Fractal Image Compression of Satellite Imageries", International Journal of Computer Applications, Vol. 30, No.3, pp. 33-36, September 2011.

[14] R.L. Yadava, M. Ram, S. Das, "Multiband Triangular Fractal Antenna for Mobile Communications", International Journal of Engineering Science and Technology, Vol. 2, No. 11, pp. 6335-6348, 2010.

[15] C.M. Kanode, H. Haddad, "Software Engineering Challenges in Game Development", Sixth International Conference on Information Technology: New Generations, pp. 260-265, 2009.

[16] N. Baek, K.H. Yoo, "Providing Direct3D Features over the Desktop OpenGL", International Journal of Smart Home Vol. 5, No. 4, pp.27-38, October 2011.

[17] W. Wu, T. Hong, S. Li, J. Guo, H. Huang, Z. Mai, D. Li, "Research of the Real-time Drawing System Based on 3D Movement Tracker", Journal of Software, Vol. 6, No. 11, Nov 2011.

[18] D. Fang, "The Study of Terrain Simulation Based on Fractal", WSEAS TRANSACTIONS on COMPUTERS, Vol. 8, Issue 1, pp. 133-142, January 2009.

[19] W. Qinglin, Y. Hua, W. Yukun, "Study on 3D Terrain Mapping Method Based on Triangulation", Proceedings of the Third International Symposium on Electronic Commerce and Security Workshops (ISECS '10), pp. 18-21, China, July 2010.

[20] J. Jeon, K. Kim, S. Jung, "A Study on the Game Programming Education Based on Educational Game Engine at School", Journal of Education and Learning, Vol. 1, No. 2, pp. 282-287, 2012.

[21] A.J. Robison, "The Design is the Game: Writing Games, Teaching Writing", Computers and Composition, Vol. 25, pp. 359-370, Elsevier Inc, 2008.

[22] M. Overmars, "Teaching Computer Science Through Game Design”, IEEE Computer, pp. 81-83, Vol. 37, No. 4, 2004.

[23] M. Isard, M. Shand, A. Heirich, "Distributed rendering of interactive soft shadows", Parallel Computing, Vol. 29, pp. 311-323, Elsevier Science B.V, 2003.

[24] X. Nan, S. Dewen, "Application of Two Rendering Techniques in the Visualization of 3D Geospatial Data", 2011 International Conference on Environmental Science and Engineering (ICESE), Procedia Environmental Sciences, Vol. 12, pp. 1432-1439, Elsevier B.V, 2012.

[25] A. Ioannidou, A. Repenning, D.C. Webb, "AgentCubes: Incremental 3D end-user Development", Journal of Visual Languages and Computing, Vol. 20, pp. 236-251, Elsevier Ltd, 2009.

[26] J. Robertson, C. Howells, "Computer game design: Opportunities for successful learning", Computers \& Education, Vol. 50, pp. 559-578, Elsevier Ltd, 2007.

[27] B.B. Mandelbrot, "Fractal Geometry of Nature", W.H. Freeman, New York, 1982.

[28] S.L. Singh, S.N. Mishra, W. Sinkala, "A New Iterative Approach to Fractal Models", Commun Nonlinear Sci Numer Simulat, Vol. 17, pp. 521-529, Elsevier B.V, 2011.

[29] P. Paramanathan, R. Uthayakumar, "Fractal Interpolation on the Koch Curve", Computers and Mathematics with Applications, Vol. 59, pp. 3229-3233, Elsevier Ltd, 2010.

[30] H.O. Peitgen, H. Jurgens, D. Saupe, “Chaos and Fractals”, pp. 87-90, Springer, New York, 1992.

\section{Authors}


International Journal of Computer Graphics \& Animation (IJCGA) Vol.4, No.1, January 2014

Farhad Soleimanian Gharehchopogh is currently Ph.D. candidate in department of computer engineering at Hacettepe University, Ankara, Turkey. And he works an honour lecture in computer engineering department, science and research and Urmia branches, Islamic Azad University, West Azerbaijan, Iran. He is a member of editorial board and review board in many international journals and international Conferences. His interested research areas are in the Operating Systems, Software Cost Estimation, Data Mining and Machine Learning techniques and Natural Language Processing. For more information please visit www.soleimanian.net

Isa Maleki is a Lecturer and Member of The Research Committee of The Department of Computer Engineering, Dehdasht Branch, Islamic Azad University, Dehdasht, Iran. He Also Has Research Collaboration with Dehdasht Universities Research Association NGO. He is a Member of Review Board in Several National Conferences. His Interested Research Areas Are in the Software Cost Estimation, Machine Learning, Data Mining, Optimization and Artificial Intelligence.

Sahar Sadouni is a M.Sc. Student in Department of Computer Engineering, Science and Research Branch, Islamic Azad University, West Azerbaijan, Iran. Her Interested Research Areas Are Computer Games, Computer Animation, Data Mining and Machine Learning.
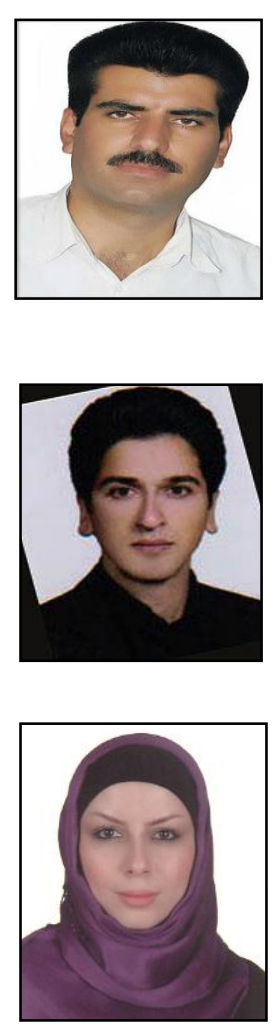